\title{
Eucation à la citoyenneté et éducation multiculturelle: Principales tendances et débats anglophones
}

\section{Abdel-Jalil Akkari}

A partir des recherches anglophones des années 90, cet article analyse les liens entre éducation à la citoyenneté et éducation multiculturelle. Nous présenterons d'abord quelques éléments sur les racines institutionnelles de l'éducation à la citoyenneté et de l'éducation multiculturelle dans les pays anglo-saxons. Nous présenterons ensuite un bilan des recherches sur l'éducation à la citoyenneté. Nous aborderons d'une manière plus détaillée deux thèmes des recherches anglophones: les différents types d'éducation à la citoyenneté et les revendications des minorités culturelles. Finalement, nous tenterons d'examiner les possibilités d'une convergence entre l'éducation multiculturelle et l'éducation la citoyenneté.

\section{Introduction}

Dans le débat éducatif, l'éducation à la citoyenneté et l'éducation multiculturelle ont un sens parfois flou, souvent prescriptif, toujours historique et contextualisé. Depuis plusieurs années, l'attention des chercheurs en éducation s'est portée sur les liens entre ces deux domaines de l'éducation. Pour traiter cette question, nous allons examiner la documentation scientifique anglophone des années 90. Cette note de synthèse n'a pas pour ambition de fournir une liste exhaustive de l'ensemble des travaux réalisés dans ce domaine mais plutôt de mettre en évidence les résultats marquants et les possibilités de convergence.

\section{Les racines institutionnelles de l'éducation à}

La citoyenneté

Il n'est guère original de rappeler ici que l'histoire de la citoyenneté est relativement récente et qu'elle a été façonnée par l'histoire particulière de chaque Etat. De nombreux groupes sociaux et peuples en étaient exclus. La déclaration universelle des droits de l'Homme, adoptée en 1948 par l'assemblée générale des 
Nations unies, aurait pu marquer le début d'une citoyenneté universelle, élargie à tous. Mais, l'organisation au sein de laquelle cette déclaration a été adoptée est basée sur l'adhésion d'Etats-Nations ayant un pouvoir inégal sur les plans politique, économique, militaire et technologique. Souvent, ces Etats-Nations, très hétérogènes sur le plan culturel, se présentent comme monoculturels et homogènes. Par ailleurs, cette déclaration est principalement inspirée par les pouvoirs occidentaux de l'après Deuxième Guerre mondiale. Elle est le produit de la philosophie et de l'orientation culturelle occidentales (Donnelly, 1984). Elle reflète les concepts de droit et de liberté individuels qui ont progressivement prévalu en occident à partir de la période des Lumières. En privilégiant les notions de responsabilité et de communauté, certaines cultures non occidentales conçoivent les relations humaines généralement plus en termes d'obligations qu'en termes de droits. Ainsi, Mercredi et Turpel (1993) observent que les amérindiens considèrent les droits comme une responsabilité collective plutôt que des droits individuels et qu'ils croient en la possibilité de développer l'autonomie individuelle sans pour autant sacrifier le sens de la communauté.

Il semble donc qu'une prise en compte de la composition culturelle diverse de la communauté internationale devrait se traduire par un élargissement des racines culturelles de la Déclaration universelle des Droits de l'Homme. Par ailleurs, si l'Etat de droit est une condition institutionnelle nécessaire pour l'exercice de la citoyenneté, elle n'est de loin pas suffisante. Bigelow (1999) observe qu'aux Etats-Unis, la constitution américaine a été un moyen légal pour préserver la stratification raciale et sociale et pour maintenir les privilèges des groupes sociaux dominants. En 1857, la cour suprême a par exemple estimé qu'un esclave ne peut pas porter plainte pour récupérer sa liberté parce qu'il est une propriété et non pas un être humain. De la reconstruction après la Guerre Civile à la période actuelle en passant par l'épopée des droits civiques l'histoire de ce pays est marquée par les immenses inégalités liées à l'esclavagisme et par le combat permanent et toujours d'actualité des minorités pour l'égalité des droits. Les indiens d'Amérique du Nord ont connu la même négation historique de leur citoyenneté (Brown, 1970).

L'examen de l'histoire de la plupart des systèmes éducatifs démontre que les liens entre éducation et citoyenneté apparaissent évidents. L'Etat a un intérêt manifeste dans le processus d'éducation. Le rôle de l'école dans la création d'un sentiment d'appartenance nationale est bien documenté dans les recherches historiques (Heater, 1990; Osborne, 1996). C'est donc l'Etat-Nation qui a donné forme, tout d'abord à l'éducation civique, ensuite à l'éducation à la citoyenneté et qui leur conferent une mission d'homogénéisation culturelle. Dans les sociétés contemporaines traversées par des conflits entre différents groupes ethnoculturels, la volonté d'homogénéisation de l'Etat apparait à la fois comme plus difficile et désirable. En effet, les revendications conjuguées des minorités régionales (de composante interne) et des minorités allogènes (d'apport externe) 
remettent à l'ordre du jour l'éducation à la citoyenneté comme une nécessité absolue, parfois même comme la solution magique.

Même si des exceptions existent comme l'interactionnisme de Dewey, l'intérêt d'émancipation de Habermas ou le dialogue chez Freire, la principale proposition pédagogique de l'éducation à la citoyenneté a été la consolidation et la perpétuation de l'idéologie de l'Etat-nation plutôt que sa contestation ou une volonté réelle de la changer (Griffith, 1998). Il faut rappeler à ce propos que l'apogée du nationalisme et de la citoyenneté est également celui de l'Etat-Nation. Mais, il faut observer que la thèse la plus plausible est celle qui stipule que c'est bien le nationalisme qui crée la nation et non pas l'inverse (Gellner, 1989).

Pour résumer, nous pouvons dire que les racines institutionnelles de l'éducation à la citoyenneté renvoie au rôle central pris par l'Etat à l'instauration de l'école obligatoire pour tous à la fin du 19ème siècle. A l'époque, l'éducation à la citoyenneté était perçue comme une opportunité de préparer les futur citoyens à une pleine participation aux institutions politiques. Aussi bien Green (1990) que Boli (1989) ont clairement montré que les intentions derrière l'instauration de la scolarité obligatoire étaient de l'ordre de l'émancipation du citoyen mais aussi de la consolidation de l'Etat. Les effets de la scolarisation de masse au niveau local ont ainsi largement contribué à la naissance d'une société civile.

L'émergence de l'éducation multiculturelle aux Etats-Unis et dans les autres pays anglophones n'a pas été soutenu par un processus similaire à celui de l'éducation à la citoyenneté. En effet, l'éducation multiculturelle est issue de la pression des organisations de défense des droits des minorités. Cette pression a permis dans un second temps les restructurations institutionnelles nécessaires à la prise en compte des discriminations dont souffrent les groupes minoritaires.

Pour schématiser, nous pouvons dire que, même si le rôle de l'Etat a été déterminant dans l'émergence des deux concepts dans les pays anglophones, l'éducation à la citoyenneté a été le produit d'un processus top-down alors que l'éducation multiculturelle est issue d'une démarche de terrain (down-top). Pour l'éducation à la citoyenneté, la question centrale est: comment homogénéiser la culture des citoyens d'un Etat- Nation. Pour l'éducation multiculturelle, la question cruciale est comment avoir une éducation publique dans laquelle se retrouvent tous les groupes culturels.

\section{Les recherche sur l'éducation à la citoyenneté}

Les recherches anglophones sur l'éducation à la citoyenneté sont caractérisées par une hétérogénéité extrême. Les recherches empiriques sur le terrain scolaire pour mesurer l'impact de l'éducation à la citoyenneté auprès des élèves ou des enseignants sont plutôt rares (Hahn, 1999). Une partie importante de ces recherches s'attache à la description et l'analyse du matériel didactique utilisé pour promouvoir l'éducation à la citoyenneté en classe. 
La rareté de la recherche empirique et la sur-abondance de la recherche spéculative et réflexive dans le domaine de l'éducation à la citoyenneté ont été constatées par plusieurs auteurs (Corbett, 1991; Zellman, 1975). Patrick et Hoge (1991) affirment sans détour que la recherche sur l'éducation à la citoyenneté offre une aide très limitée aux enseignants et qu'elle a besoin d'une amélioration notable. Sears (1996) va dans le même sens en appelant à une clarification conceptuelle. Parker (1990, p. 18) estime même que l'«éducation à la citoyenneté est si vague et globalisante qu'elle peut signifier n'importe quoi pour n'importe qui».

De nombreux travaux de recherche anglophones soulignent l'importance des facteurs tels que climat de l'école et climat de la classe. En effet, tout en reconnaissant que l'école et la classe ne sont pas et ne peuvent pas être une démocratie, de nombreux chercheurs ont identifié un niveau élevé de valeurs démocratiques chez les élèves fréquentant des écoles à environnement démocratique. Les élèves qui sont moins autoritaires, plus tolérant et plus optimistes par rapport aux processus démocratiques sont présents dans les écoles caractérisées par une structure organisationnelle démocratique dans laquelle les directeurs orientent vers une direction pédagogique cohérente mais laissent une large autonomie aux enseignants. Ces écoles permettent la participation des élèves dans les processus décisionnels qui les affectent (Angell, 1991; Boyer 1990).

Harwood (1992) a analysé 12 études portant sur le climat de classe. Elle a constaté que les interactions en classe influencent les représentations et les attitudes politiques des élèves. Un climat d'ouverture dans la classe, caractérisé par la participation des élèves et la libre expression a un effet positif sur l'attitude des élèves envers la connaissance du politique et la participation politique.

Van Sledright et Grant (1994) observent que l'éducation à la citoyenneté est difficile à enseigner. Des conflits et des contradictions apparaissent à partir de trois dilemmes de l'enseignant: la préparation du curriculum, l'exercice de l'autorité et la gestion de la classe. Les enseignants font face aux problèmes suivants: (1) le pouvoir que l'enseignant accepte de déléguer aux élèves sur le choix du contenu et sur la forme d'enseignement, (2) le respect des directives curriculaires officielles, (3) l'accomplissement de l'ensemble du programme et (4) la participation des élèves et la gestion du temps.

Dunkin, Welch, Merritt, Phillips et Craven (1998) ont identifié sept catégories majeures qui déterminent le savoir pratique des enseignants à propos de l'éducation à la citoyenneté: (1) connaissance du contenu et des ressources; (2) connaissance des élèves; (3) connaissances pédagogiques; (4) connaissance du contexte communautaire; (5) gestion du temps; (6) effets affectifs et (7) contrôle et discipline dans la classe. On observera donc que la recherche met en évidence le déséquilibre entre le nombre de catégories favorables à l'éducation à la citoyenneté et la capacité de l'enseignant d'y répondre. Ce déséquilibre est une source de tension permanente dans gestion quotidienne de la classe.

Constatant que les attitudes favorables à la citoyenneté se stabilisent vers 9-10 ans, Womack et King (1982) recommandent une introduction précoce de l'édu- 
cation à la citoyenneté dans les programmes scolaires. Cette place à accorder à l'éducation à la citoyenneté dans le curriculum fait d'ailleurs l'objet de multiples études et controverses (National Council for Social Studies, 1992; National Curriculum Council, 1990; New York State Social Studies Syllabus Review and Development Committee, 1991; Qualifications and Curriculum Authority, 1998).

Pour Griffith (1998), les finalités de l'éducation pour la citoyenneté (education for citizenship) a oscillé dans les années 90 entre deux images qui ont eu une position influente dans la construction des programmes scolaires (National Curriculum). D'une part, une image décrivant des enfants confiants réclamant leur place comme décideurs dans une culture de haute modernité. Autonomes, mais moralement bien dotés, ces enfants seront les citoyens du 21 ème siècle. Les mots qui accompagnent cette vision optimiste sont: capacité, empowerment, sensibilité sociale, coopération, recherche. D'autre part, une image centrée sur des enfants apprenant les contenus scolaires. Il s'agit là d'une vision nostalgique ayant plus à voir avec le 19ème siècle. Des écoliers en uniforme parfaitement alignés écoutant religieusement une présentation didactique dispensée par un enseignant «autoritaire». Les mots qui recouvrent cette deuxième image sont: standards, rigueur, discipline, saine compétition, traditions, retour aux connaissances de base (backto-basics), tests. Cette polarisation du débat démocratique et pédagogique au sein même du groupe majoritaire ne facilite pas la prise en compte des revendications minoritaires. Ces dernières sont souvent perçues comme secondaires.

En Australie, on retrouve les mêmes discussions sur l'introduction de l'éducation à la citoyenneté dans le curriculum. Ce pays, comme d'autres pays d'immigration, doit affronter la difficile tâche de combiner diversité culturelle et unité. L'approche de l'éducation civique et l'éducation à la citoyenneté (Civic and citizenship education) a été introduite en Australie à travers des expériences d'apprentissage qui préparent les élèves à l'exercice effectif de la citoyenneté et l'habitus d'une identité multiculturelle et d'une société démocratique (O'Brien \& Parry, 2001; Torney-Purta, Schwille \& Amadeo, 1999). Le déclin de l'éducation civique et les inquiétudes concernant l'avenir de l'éducation à la fin des années 80 a donné naissance en Australie au Ministerial Council on Education, Employment, Training and Youth Affairs [MCEETYA]. L'objectif principal de cet organisme est de développer un ensemble d'objectifs nationaux pour l'enseignement. Le MCEETYA a proposé que l'éducation à la citoyenneté joue un rôle essentiel dans ce processus. Selon le MCEETYA (1999), les élèves doivent avoir à la sortie de l'école la capacité:

- de jugement et de responsabilité dans les domaines de la morale, de l'éthique et de la justice sociale,

- de donner un sens à leur monde et de penser et réfléchir sur les différents problèmes qui les concernent,

- de prendre des décisions rationnelles et appropriées à propos de leur vie,

- d'assumer la responsabilité de leurs propres actions,

- d'être des citoyens actifs et informés du fonctionnement de leur système politique. 
Concernant la place de l'éducation à la citoyenneté dans le curriculum en Australie, l'éducation civique ou l'éducation à la citoyenneté ne sont pas présentes en tant que discipline particulière mais font partie des enseignements consacrés aux études de la société et de l'environnement (Studies of Society and Environnement Syllabus, The Human Society and its Environment Syllabus).

Le Civics Expert Group (1994) a proposé l'inclusion dans le curriculum national de diverses activités liées à l'éducation à la citoyenneté et l'éducation multiculturelle: l'héritage de l'Australie, la composition multiculturelle de la population, la participation active des citoyens à la vie démocratique, le rôle des relations internationales, les valeurs clefs dans la société démocratique, le processus démocratique et le développement durable.

En dépit de la rhétorique sur l'importance du multiculturalisme dans la nouvelle identité australienne, l'articulation des différents idéaux et valeurs présents dans les nombreux groupes ethniques et culturels est une tâche difficile. En effet, si la société australienne est ancrée dans un modèle inclusif de la citoyenneté dans lequel cette dernière est à la fois un statut et un ensemble de droits de tous les habitants, la persistance des questions de la discrimination et de l'inégalité sociale montre le caractère théorique de l'inclusion.

De nombreux travaux anglophones de recherche sur l'éducation à la citoyenneté se focalisent sur deux objets: la gestion des relations à l'intérieur du système scolaire (climat de classe, climat d'établissement) et la place de l'éducation à la citoyenneté dans le curriculum. Dans la suite de cet article, nous allons nous centrer sur les travaux qui permettent le mieux d'aborder les liens entre éducation à la citoyenneté et éducation multiculturelle.

\section{Les différents types d'éducation à la citoyenneté}

L'idée que la vie à l'école doit être une préparation à la vie dans la société est un aspect bien documenté dans la littérature pédagogique en commençant par le rôle précurseur de Dewey. Les notions d'activité, droits et responsabilités sont au centre du mouvement de l'éducation nouvelle (Dewey, 1916).

L'analyse de l'éducation à la citoyenneté dans le curriculum a permis à Sears et Hughes (1996) d'identifier une série d'approches que l'on peut situer entre une approche passive à une approche active. Pour schématiser, on peut dire que la première est centrée sur les connaissances et la deuxième sur les expériences. L'approche passive est centrée sur la socialisation et le développement d'une loyauté envers l'Etat-Nation. Le plus souvent, cette approche est traduite dans le curriculum par l'accumulation de connaissances statiques et limitées de l'histoire et des traditions culturelles nationales. L'approche active met en exergue l'engagement quotidien de l'apprenant et sa participation dans la transformation des contextes local, national et même international (Sears, 1994). 
En Grande-Bretagne, Griffith (1998) distingue également deux approches de l'éducation à la citoyenneté: apprendre sur la citoyenneté et apprendre par (dans) la citoyenneté (learning about citizenship and learning in citizenship). Apprendre sur la citoyenneté consiste à enseigner aux élèves leurs futurs droits et devoirs quand ils deviendront adultes. Apprendre par et dans la citoyenneté est centré sur l'élève et vise à développer le sens de la citoyenneté par l'exploration des devoirs et des responsabilités actuels des jeunes à l'école. Un apprentissage actif serait la meilleure préparation pour une citoyenneté active dans une démocratie (Starkey, 1991).

Une stratégie habituellement utilisée pour apprendre sur la citoyenneté consiste à utiliser en classe un matériel didactique diversifié: brochures descriptives, jeux de rôle, etc. ... Certains auteurs sont très enthousiastes à propos de cette démarche qui tente à travers des activités souvent ludiques d'intéresser les enfants aux droits de l'homme et à la citoyenneté. L'utilisation des jeux de rôles et de simulations sur la citoyenneté dans la formation des enseignants est une démarche très pertinente. De nombreux outils disponibles pour apprendre sur la citoyenneté sont disponibles sur support informatique comme par exemple Carrigan street, Project Space, the Best Days Of Our lives (Davison, 1987; Scetlander, 1986; Starkey, 1991).

Toutefois, Griffith (1998) se montre très réservé envers cette approche didactique qui postule que l'élève manque de motivations pour l'éducation à la citoyenneté ou l'éducation aux droits de l'homme qui seraient trop éloignées de sa réalité présente. Une autre objection récurrente envers cette démarche didactique est son optimisme. En effet, il est légitime de se demander comment des enseignants armés par leur seul matériel didactique/ludique pourraient réussir là où les politiciens et les autres instances de socialisation ont échoué: c'est à dire développer un sens civique actif chez les jeunes. Comme le rappelle ironiquement Kukathas (2000), un régime politique libéral ne dépend pas de l'éducation civique.

La deuxième approche consiste à apprendre par et dans la citoyenneté à l'intérieur même de l'école en appliquant et en discutant les concepts de base de la citoyenneté: justice, égalité, discrimination, liberté et auto-détermination (Han, 1998; Morse, 1993). Ces concepts doivent être enseignés en se référant aux propres expériences des élèves à l'intérieur même de l'école. Les activités entreprises dans cette perspective sont généralement extra-curriculaires: conseil $\mathrm{d}$ de classe ou d'école, recherche de fonds, activités caritatives. Ces activités permettent d'atteindre des objectifs précis et de résoudre des problèmes concrets (Starkey, 1991; Heater, 1990). Cette deuxième approche a également subi de nombreuses critiques. L'apprentissage de la citoyenneté par des actions à l'intérieur de l'école ou de la classe est considéré par certains auteurs comme un «saupoudrage démocratique» (Hart, 1992).

Plus important encore, l'apparente démocratisation de la vie scolaire s'effectue généralement à travers la présence de représentants des élèves dans les différents organes scolaires. Les droits des élèves sont ainsi exercés d'une manière indirecte 
et non pas à travers les décisions et les actions personnelles de chaque élève. Les élèves exercent des privilèges accordés et non pas des droits (Franklin, 1995). Les enseignants et les autres adultes bénéficient clairement de droits différents que ceux accordés aux enfants dans un système caractérisé par un accès différencié à la citoyenneté. La citoyenneté factice accordée aux élèves ne serait alors pas payante sur le plan pédagogique.

D'un point de vue plus global, toutes les institutions modernes y compris l'école semblent laisser de coté la possibilité d'émancipation en développant plutôt des mécanismes de domestication (Giddens, 1991). En dépit de tous les efforts qu'elle déploie, l'école n'est selon Bernstein que le relais d'un pouvoir qui la dépasse:

Education is a relay for power external to it [...]. The education system's pedagogic communication is simply a relay for something other than itself. Pedagogic communication in the school, in the nursery, in the home, is the relay for class relations; the relay for gender relations, the relay for religious relations, for regional relations. Pedagogic communication is the relay for patterns of dominance external to it-self (Bernstein, 1990, pp. 168-169).

Il paraît clair que la citoyenneté est aujourd'hui à la recherche de sa place à l'école. Ni l'approche visant à développer des connaissances (learning about citizenship), ni celle qui vise à l'émergence de compétences en éducation à la citoyenneté (learning in citizenship) ne semblent faire l'unanimité des observateurs anglophones. Même la question technique qui concerne la place de l'éducation à la citoyenneté dans le curriculum donne lieu à un débat animé. Pour certains, l'éducation à la citoyenneté doit être un élément du curriculum enseigné dans une ou plusieurs disciplines. Pour d'autres, elle doit rester une activité extra-curriculaire ou transversale (Edwards \& Fogelman, 1993).

De surcroît, la prise en compte de la diversité culturelle des élèves complique la tâche des enseignants intéressés par l'éducation à la citoyenneté. S’ils optent pour un apprentissage sur la citoyenneté, ils se heurtent à une difficulté redoutable: comment aborder la conception occidentale de la citoyenneté sans pour autant oublier que l'Occident n'a jamais fait bénéficier de la démocratie les peuples qu'il a colonisé?

Si les enseignants choisissent un enseignement de la démocratie par l'exercice de citoyenneté à l'école, ils se heurtent au fait que l'école est une institution reproductrice des inégalités sociales et culturelles. Les groupes minoritaires ont un pouvoir et un contrôle réduit sur les institutions éducatives qui accueillent leurs enfants (Ogbu, 1987).

La diversité des pratiques de l'éducation à la citoyenneté ne permet pas de brosser une typologie exhaustive. Toutefois, Akkari (sous presse) a mis en évidence six composantes principales de la citoyenneté qui peuvent se situer entre un niveau minimal et un niveau maximal. Sur le plan politique et institutionnel, le niveau minimal se situe au niveau des garanties constitutionnelles de l'exercice de la ci- 
toyenneté (accordées habituellement aux nationaux) et le niveau maximal exprime la participation de tous les groupes socioculturels à la prise de décision politique. Sur le plan culturel, le niveau minimal exprime le respect des identités culturelles tandis que niveau maximal ouvre la possibilité d'une citoyenneté multiculturelle, voir transculturelle, dans laquelle les individus et les groupes traversent et transforment leurs identités culturelles d'origine. Sur le plan éducatif/pédagogique, le niveau minimal exprime la nécessité d'une garantie d'accès aux différents niveaux du système éducatif et le niveau maximal renvoie à l'égalité des résultats. Les différentes conceptions de l'éducation à la citoyenneté vont habituellement se rapprocher de l'une ou l'autre de ces composantes. A titre d'exemple, un enseignant qui privilégie la composante (1) (cf. tableau 1) va donner la priorité à des activités qui développent chez les élèves les connaissances de l'organisation politique et législative du pays.

Tableau 1: Composantes principales de la citoyenneté; Adapté de Akkari (2002)

\begin{tabular}{|l|l|l|l|}
\hline & Institutionnel et politique & \multicolumn{1}{|c|}{ Culturel } & \multicolumn{1}{|c|}{ Educatif/pédagogique } \\
\hline Minimal & $\begin{array}{l}\text { (1) Droits et obligations } \\
\text { liés à l'identité nationale }\end{array}$ & $\begin{array}{l}\text { (3) Identité cul- } \\
\text { turelle }\end{array}$ & $\begin{array}{l}\text { (5) Accès au système éducatif } \\
\text { et traitement équitable de tous } \\
\text { les groupes }\end{array}$ \\
\hline Maximal & $\begin{array}{l}\text { (2) Inclusion sociale et } \\
\text { participation politique }\end{array}$ & $\begin{array}{l}\text { (4) Citoyenneté } \\
\text { multiculturelle }\end{array}$ & $\begin{array}{l}\text { (6) Appropriation des savoirs } \\
\text { scolaires, développement de la } \\
\text { pensée critique et égalité des } \\
\text { résultats }\end{array}$ \\
\hline
\end{tabular}

Cette tentative de typologie des différentes composantes de la citoyenneté montre la difficulté de parvenir à un consensus à propos de la place et le type d'éducation à la citoyenneté à développer dans la société et à l'école. Les travaux de Gikandi (1996) et de Phillips (1996) illustrent par exemple la difficulté de parvenir à définir les identités nationales (citoyennetés) anglaises ou galloises actuelles dans un contexte marqué par l'immigration massive et la disparition de l'empire Britannique.

\section{Revendications des minorités et remise en ques- tion du multiculturalisme}

Les promoteurs de l'éducation à la citoyenneté et de l'éducation multiculturelle se heurtent à la nécessité et à la difficulté de gérer les revendications minoritaires. Le débat à propos des valeurs à transmettre à l'école est une occasion habituellement utilisée pour aborder ces revendications. Comme le rappelle Torres (1998), les théories de la citoyenneté ont été élaborées dans la tradition politique occidentale par les mâles hétérosexuels blancs. Certains groupes sociaux ont été systé- 
matiquement exclus de l'accès à la citoyenneté (Cairns, Courtney, MacKinnon, Michelmann \& Smith, 1999). Dans le contexte colonial en particulier, le sujet indigène n'a jamais été reconnu comme citoyen à part entière.

Dans plusieurs pays anglophones le débat sur les valeurs qui sous-tendent l'éducation à la citoyenneté a été vif ces dernières années (Barber, 2000). En Grande-Bretagne, il y a eu un net virage avec l'alternance politique au pouvoir. Sous les conservateurs, les discussions portaient sur l'enseignement du christianisme et la préservation de l'héritage chrétien. Avec l'arrivée au pouvoir du «New Labour», la priorité est placée dans une éducation à citoyenneté basée sur la connaissance et la valorisation de notre tradition occidentale de liberté politique (Crick, 1998). Les objectifs de l'éducation à la citoyenneté dans le contexte anglais seraient de permettre aux enseignants de jouer un rôle crucial dans la transmission de principes éthiques en absence de Dieu et de Marx.

Aux États-Unis, c'est le débat sur l'éducation multiculturelle qui a mobilisé les intellectuels autour de la question des valeurs. Il s'est structuré durant les dernières décennies autour d'un axe allant de l'empowerment des groupes opprimés à la création d'une unité nationale par l'enseignement de valeurs culturelles communes. A l'origine, les tenants de l'éducation multiculturelle comme Banks (1993, 1999), Sleeter (1991), Grant et Ladson-Billings (1997) et bien d'autres étaient préoccupés par la valorisation des groupes opprimés. L'intégration de leur histoire et de leurs cultures dans le curriculum des écoles publiques et dans les manuels scolaires était l'objectif pédagogique prioritaire. En général, ces auteurs visaient aussi la réduction des discriminations, l'élimination du sexisme et l'égalité des chances éducatives.

Dès la fin des années 80 , une réaction violente contre l'éducation multiculturelle était initiée par les milieux conservateurs. Elle soutenait une éducation centrée autour des valeurs des groupes dominants. Ce mouvement a pris deux formes. La première proposait l'unification et l'assimilation des élèves autour d'un ensemble de valeurs ayant pour sources les traditions WASP (blanches anglo-saxonnes protestantes). D'autres auteurs soutenaient l'unification des élèves autour un corps de savoir culturel (body of culture knowledge) issu de l'examen de la société américaine contemporaine (Hirsch, 1987). Ces deux branches du mouvement conservateur dans le domaine de l'éducation s'inscrivent dans une perspective pédagogique plus vaste, celle du retour à l'essentiel (back-to-basics) qui prône à la fois le retour des connaissances traditionnelles et surtout de celles issues de l'héritage WASP dominant.

Dès lors, le multiculturalisme est jugé très dangereux pour l'unité et la tradition politique des Etats-Unis. Schlesinger (1991), auteur de plusieurs livres sur l'histoire des Etats-Unis, estime par exemple que les institutions et la culture du pays sont principalement issues des valeurs anglaises et européennes et que ce noyau de valeurs doit être la seule source de l'unité nationale et le pilier de la citoyenneté. Selon Schlesinger, la tradition anglo-saxonne a été durant deux siècles et le reste encore dans une large mesure l'influence dominante sur la culture et la 
société américaine. Cette tradition WASP venant d'Angleterre avait fixé la langue de la nouvelle nation, ses lois, ses institutions, ses idées politiques, ses préceptes, ses prières. En d'autres termes, la tradition WASP avait fixé les modalités d'accès et d'exercice de la citoyenneté

Hirsch (1991) a développé pour sa part le concept d'alphabétisation culturelle (cultural literacy) qui concerne une période historique particulière. Selon Hirsch, le savoir culturel requis en 1990 est différent de celui de 1890. Ce savoir doit être issu de travaux de recherche. Il a ainsi identifié 5000 noms, phrases, dates et concepts prioritaires. Pour les enfants appartenant a des groupes minoritaires, Hirsch souligne l'importance de leur enseigner cette culture de base. Il n'envisage l'enseignement d'autres valeurs culturelles que si elles n'entrent pas en contradiction ou en compétition avec la culture dominante des Etats-Unis (Hirsch, 1991). Il s'agit pour lui de sélectionner des valeurs non-conflictuelles compatibles avec la culture WASP.

La préoccupation de l'unité culturelle aux Etats-Unis est d'autant plus polémique que les projections démographiques indiquent clairement que la composition ethnique du pays est dans un processus de changement rapide et irréversible. Les élèves appartenant à des groupes minoritaires représenteront $46 \%$ des effectifs en 2020 alors qu'ils ne représentaient que 27\% des effectifs en 1982 (Pallas, Natriello \& McDillet, 1989). Par ailleurs, 90\% des nouveaux immigrants aux Etats-Unis viennent de l'extérieur de l'Europe (U.S. Bureau of the Census, 1994). Suite aux événements tragiques du 11 septembre aux Etats-Unis, de nombreux éditeurs scolaires ont précipitamment entrepris des modifications de manuels scolaires pour relayer les préoccupations du moment. Ainsi, l'éditeur Prentice-Hall's va changer la conclusion de l'un des manuels d'histoire le plus utilisé à l'école secondaire en substituant les passages vantant la diversité par des passages focalisés sur la défense de la liberté. L'idée n'est pas de revenir à l'ancienne formule «nous sommes tous les mêmes» mais d'insister sur l'appartenance à la même nation ("we're all Americans.», "a nation of many becomes a nation of one») (Gordon, 2002).

Au Canada, Gwyn (1995) a une analyse assez proche de Schlesinger puisqu'il s'inquiète du recul du sens du destin commun. Il estime que le Canada est plus vulnérable que les autres pays anglophones à la fois à cause de ses liens étroits avec les Etats-Unis, mais aussi en raison du développement du nationalisme francophone au Québec. Le philosophe Charles Taylor (1992) a articulé son travail sur l'analyse de ce que veut dire être canadien. Le problème central pour la citoyenneté canadienne est, selon cet auteur, la place du Québec dans la confédération canadienne à laquelle viennent s'ajouter les revendications d'autodétermination des peuples premiers. L'histoire de l'éducation à la citoyenneté au Canada montre un passage d'une phase au début du siècle où une vision assimilationniste dominait à un abandon actuel de l'éducation à la citoyenneté dans une politique éducative où la préparation à l'emploi domine (Osborne, 1996). Kaplan (1993) estime que l'échec passé de l'éducation canadienne à la citoyenneté et son pro- 
bable échec dans le futur sont inéluctables si aucune mesure n'est prise pour reconceptualiser l'éducation à la citoyenneté à la lumière des changements démographiques, culturels, politiques et sociaux qu'a connu le pays.

Aussi bien aux Etats-Unis qu'au Canada, c'est la gestion des différences culturelles qui cristallisent les débats sur l'éducation à la citoyenneté et l'éducation multiculturelle. Comme le rappellent de nombreux spécialistes de l'éducation multiculturelle, le défi est de considérer qu'être différent n'est pas être déficient (Mehan, Lintz, Okamoto \& Eills, 1995; Tiedt \& Tiedt, 1990). La montée des revendications minoritaires provoque un débat salutaire sur le rôle de la citoyenneté dans la viabilité des sociétés multiculturelles. Autrement dit, quel rôle une re-conceptualisation de la citoyenneté peut-elle jouer dans la recherche des moyens de vivre ensemble avec nos différences, nos contentieux historiques, voire nos divergences actuelles (Macedo, 2000). La politique de discrimination positive a été l'une des réponses institutionnelles controversées utilisées pour gérer les différences culturelles.

Ecartant tout risque sérieux lié au multiculturalisme, Saïd (1993) estime que le multiculturalisme met en lumière la nécessité de changements politiques et un abandon de la manière dont les femmes, les minorités ethniques et les migrants récents sont traités. Il rappelle à juste titre que sur le plan historique, les processus de revendication et d'émancipation des minorités dans leurs formes les plus puissantes sont des tentatives d'intégration et non pas de séparation. Il s'agit d'histoires de vie de personnes qui ont été exclues par le groupe dominant et qui se sont mobilisées pour avoir une place. Si les représentations traditionnelles du groupe dominant ne sont pas suffisamment généreuses et flexibles pour admettre de nouveaux groupes, alors le mieux à faire est de changer ces représentations plutôt que de rejeter la menace supposée des groupes émergeants sur la cohésion nationale.

Dans l'ensemble des pays anglophones est apparu ces dernières années l'expression «citoyenneté multiculturelle» qui vise à reconstruire le sens de l'identité nationale. Les directives curriculaires cherchent par exemple à célébrer la diversité culturelle tout en appelant à l'idéal de la cohésion sociale et nationale. Toutefois, la vision assimilationniste n'a pas disparu en raison du développement des partis d'extrême droite comme le "One Nation Party» en Australie ou le mouvement militant pour l'anglais seulement aux Etats-Unis.

Faist (2000) estime que la citoyenneté a été historiquement liée à l'évolution des Etats-Nations. Il postule l'existence de trois types de citoyenneté: une $c i$ toyenneté nationale, une citoyenneté multiculturelle et une citoyenneté transnationale. La citoyenneté nationale est unitaire et enracinée dans une seule culture politique. La citoyenneté multiculturelle exige la reconnaissance par la culture politique des différences culturelles. La citoyenneté transnationale articule des éléments de cultures politiques provenant de plusieurs états. Faist estime que le syncrétisme transnational en matière de citoyenneté permet de dépasser la conception statique de la culture comme conteneur. 
Print (1996) estime qu'une perspective multiple de la citoyenneté devait être basée sur les différents rôles que les individus jouent dans la vie civique. Cette approche constitue un virage dans l'éducation à la citoyenneté en amenant le traitement explicite des valeurs. La possibilité est ainsi donnée à chaque individu de réfléchir sur la diversité de ses valeurs. Banks (2001) parle de son côté de «citoyenneté multiculturelle» qui exige de chaque individu de se situer et de se positionner par rapport aux contextes local, culturel, national et international. Au Canada, Kymlicka (1995a, 1995b) et Kymlicka \& Norman (2000) proposent également le concept de «citoyenneté multiculturelle». On trouve d'autres expressions comme «Citoyenneté multiple» ou "citoyenneté globale» (Giddens, 1991; White, 1991). Toutes ces tentatives de re-conceptualisation de la citoyenneté et par voie de conséquence de l'éducation à la citoyenneté démontrent la convergence dans les pays anglophones entre l'éducation à la citoyenneté et l'éducation multiculturelle.

La faisabilité pédagogique du concept de "citoyenneté multiculturelle» a été abordée par Webber (1996) qui estime que la théorie et la pratique de la citoyenneté multiculturelle pose la question difficile de la réconciliation entre unité et diversité culturelle. Selon cet auteur, la citoyenneté englobe obligatoirement un sentiment d'unité de d'identité. Or, le multiculturalisme reconnaît, encourage et célèbre la différence et la diversité. Inévitablement le conflit peut surgir entre ces deux points d'ancrage de la citoyenneté multiculturelle. Il est alors important de répondre à la question suivante: jusqu'à quel point la diversité est elle consistante avec la citoyenneté nationale? Weber explore cette question en abordant deux étude de cas. La première concerne une jeune fille d'origine musulmane qui a été suspendue de son école après avoir mis le hijab. Le deuxième cas traite de la pratique de l'excision féminine. Si ces deux situations sont peu nombreuses, elles témoignent de la nécessité d'examiner les limites du relativisme culturel.

Il n'est pas superflu de rappeler aussi que la notion d'identité nationale n'est pas appropriée pour promouvoir l'acceptation et le respect de l'autre dans une société multiculturelle. Le curriculum scolaire qui est généralement basé sur une sélection des évènements historiques ou contemporains et une interprétation limitée de ce que signifie l'appartenance à un pays ne peut plus répondre aux nécessités actuelles de l'éducation. Comme le souligne Hébert (1997), une reconceptualisation profonde de l'éducation à la citoyenneté est nécessaire:

The task has become one of elaboration of a model of citizenship education which is likely to be ale to form a citizenry respectful of multiple identities, sharing a common sense of belonging and having full parity of rights and obligations, duties and responsibilities ( $p$. 3).

Ce foisonnement de termes associant citoyenneté et multiculturalisme dans le monde anglophone sous-estime le rôle de l'Etat-nation dans la naissance et le développement de la citoyenneté abordés dans la première partie de cet article. Par conséquent, la fécondité de la notion de citoyenneté multiculturelle sur les plan 
conceptuel et pratique demeure problématique (Bissoondath, 1994; Feinberg, 1998).

\section{Les perspectives de convergence entre éducation à la citoyenneté et éducation interculturelle}

Au terme ce cette note de synthèse destinée à donner un aperçu synthétique sur la littérature anglophone, nous pouvons dire que les conceptions traditionnelles de la citoyenneté et de la multiculturalité sont largement caduques dans les sociétés anglophones contemporaines. La tendance en cours dans ces pays consiste à concevoir l'éducation à la citoyenneté dans une perspective complémentaire à celle de l'éducation multiculturelle (Lynch, 1992). Cette convergence peut prendre deux directions opposés. La première, impulsée par les tenants inconditionnels de la globalisation économique consiste à considérer l'éducation comme un sous-produit de l'économie dans un monde composé de consommateurs cosmopolites et multiculturels (Fullinwider, 2001; Mac an Ghaill, 1996; Ranson, 1993). La deuxième direction postule un rôle central de l'éducation dans la lutte contre les inégalités entres pays et à l'intérieur de chaque pays. C'est ce que propose notamment les auteurs du courant de la pédagogie critique (McLaren \& Farahmandpur, 2001).

Dans les dernières années, l'Etat-Nation comme source de support particulièrement par son rôle d'Etat-providence est remis en question à la fois par la globalisation économique, par les idées de citoyenneté européenne, citoyenneté multiculturelle ou même mondiale mais aussi par le réveil des minorités ethniques ou régionales. Haire et Manley-Casimir (2000) soulignent par exemple une évolution claire dans la conception de l'éducation à la citoyenneté au Canada où l'on est passé d'une orientation dans laquelle les élèves devraient être préparés au rôle de citoyens responsables à une posture clairement économique dans laquelle les élèves devraient être préparés à jouer leur fonction de travailleur dans une force de travail compétitive et globalisée:

This trend can be seen in the pressures exerted on individual schools and school systems to form partnerships with businesses, with a purportedly educational focus, but with underlying, product-oriented price attached. Concepts of responsible citizenship, which should be central to educational theory and practice, are displaced with school policies informed by an economic agenda ( $p$. 155).

En privilégiant un économisme étroit, la globalisation accélère la constitution d'une citoyenneté à plusieurs vitesses. Reprenant la notation financière adoptée dans les milieux internationaux de la finance, Torres (1998) parle de l'existence de deux grandes catégories de citoyens. Une catégorie de citoyens (Triple A) qui peuvent exercer toutes les possibilités de représentation et de participation politique à travers leurs votes et actions politiques. Ils sont connectés aux réseaux du pouvoir mais aussi au monde virtuel des nouvelles technologies de l'information 
et de la communication. Et une catégorie de citoyens de seconde classe (class B) qui sont marginalisés politiquement et économiquement. La survie quotidienne est leur majeure préoccupation.

Le courant de la pédagogie critique propose une autre globalisation, celle de la lutte contre les inégalités (Freire, 1998; Lister, 1990). Évidemment, la prise en compte de la dimension Nord-Sud est centrale dans toute tentative de construire une convergence féconde entre éducation multiculturelle et éducation à la citoyenneté.

Pour s'opposer à la globalisation économique avec ses trois piliers: la libéralisation, la privatisation et la déréglementation, Andrews (1991) propose une éducation dans une perspective planétaire et solidaire.

The critical future of the planet requires more then ever an ethic of solidarity, there is an urgent need to rebuild sense of community. The crucial importance of duty in this context is that implies a commitment, an obligation, for individuals themselves to solve the problems within their communities. It puts the local, and now the global, well-being at the center of citizens' concerns, above that of self-interest (p. 215).

C'est en réaffirmant la dimension politique et sociale de l'éducation à la citoyenneté et de l'éducation multiculturelle que nous pouvons leur éviter de se transformer en recettes miracles et décontextualisées. McLaren (1998) rappelle à juste titre que l'urgence n'est pas dans le respect des différences:

The conceptual net known as critical pedagogy has been cast so wide at times so cavalierly that it has come to be associated with anything dragged up out of the troubled and infested waters of educational practice, from classroom furniture organized in a dialogue friendly circle to feel-good curricula designed to increase students' self-image. Its multicultural equivalent can be linked to a politics of diversity that includes respecting difference through the celebration of "ethnic» holidays and themes such as Black history moth and Cinco de Mayo. If the term critical pedagogy is refracted onto the stage of current educational debates, we have to judge it as having been largely domesticated in a manner that many of its early exponents, such as Brazil's Paulo Freire, so strongly feared (p. 448).

Cet article nous a permis de montrer les différences dans les conditions d'émergence institutionnelle de l'éducation à la citoyenneté et de l'éducation multiculturelle dans les pays anglophones. Si l'éducation à la citoyenneté émerge comme outil de mise en œuvre de l'Etat-Nation et de ses institutions démocratiques, l'éducation interculturelle est la réponse du système éducatif aux revendications minoritaires contestant la monoculturalité du système éducatif mis en place par l'Etat-Nation. Au niveau des recherches, nous avons mis en évidence que les recherches empiriques tentent souvent de mesurer les liens entre le climat de la classe (ou de l'école) et les attitudes des élèves envers la citoyenneté. D’autres recherches essaient de déterminer la place de l'éducation à la citoyenneté dans le curriculum ou les valeurs à enseigner dans le cadre de l'éducation à la citoyenneté. Aussi bien l'éducation à la citoyenneté que l'éducation multiculturelle sup- 
posent un certain nombre de valeurs communes mais aussi et surtout la reconnaissance des rapports de force et l'engagement pour les transformer durablement.

Cet article a clairement mis en lumière que la citoyenneté et la multiculturalité sont au cœur du débat éducatif actuel partout dans le monde: celui de l'aptitude du système scolaire à socialiser mais aussi à accepter et à valoriser l'altérité.

\section{Références bibliographiques}

Akkari, A. (2002). Toward a critical perspective in citizenship education. World Studies in Education., 2, (2), 5-27.

Andrews, G. (1991). Citizenship. London: Lawrence \& Wishart.

Angell, V. (1991). Democratic climates in elementary classrooms: A Review of theory and research. Theory and Research in Social Education, 19, (3), 241-266.

Banks, J. (2001). Citizenship education and diversity: Implications for teacher education. Journal of Teacher Education, 52, (1), 5-16.

Banks, J.A. (1993). Multicultural education: Historical development, dimensions, and practice. In L. Darling-Hammond (Ed.), Review of Research in Education (pp. 3-50). Washington DC: American Educational Research Association.

Banks, J.A. (1999). An introduction to multicultural education. Boston: Allyn and Bacon.

Barber, B. (2000). Challenges to the common good in the age of globalism. Social Education, 64, (1), 8-13.

Bernstein, B. (1990). The structuring of pedagogic discourse: Volume IV, Class, Codes and control. London: Routledge.

Bigelow, B. (1999). Standards and multiculturalism. Rethinking Schools, 13, (4). [http://www.rethinkingschools.org/Archives].

Bissoondath, N. (1994). Selling illusions: the cult of multiculturalism in Canada. Toronto: Penguin.

Boli, J. (1989). New citizens for a new society: the institutional origins of mass schooling in Sweden. Oxford: Pergamon Press.

Boyer, E. L. (1990). Civic education for responsible citizens. Educational Leadership 48, (3), 4-7.

Brown, D.(1970). Bury My Heart at Wounded Knee. New York: Henry Holt and Company.

Cairns, A.C., Courtney, J.C., MacKinnon, P.; Michelmann, H.J. \& Smith, D.E. (Ed.). (1999). Citizenship, diversity, \& pluralism: Canadian and comparative perspectives. Montreal \& Kingston: McGill-Queen's University Press.

Civics Expert Group (1994). Whereas the people: civics and citizenship education. Canberra: Australian Government Printing Service.

Corbett, M. (1991). American public opinion. New York: Longman.

Crick, B. (1998). Lessons to make citizens of us all. Time Educational Supplement, 27 March.

Davison, A. (1987). The best days of our lives. New castle: Northtern Micromedia.

Dewey, J. (1916). Democracy and education. New York: Macmillan.

Donnelly, J. (1984). Cultural relatives and universal human rights. Human Rights Quarterly, $1,(4), 400-419$.

Dunkin, M.J., Welch, A., Merritt, A., Phillips, R. \& Craven, R. (1998). Teachers' explanations of classroom events: knowledge and beliefs about teaching civics and citizenship. Teaching and Teacher Education, 14, (2), 141-151.

Edwards, J. \& Fogelman, K. (1993). Developing citizenship in the curriculum. London: Fulton.

Faist, T. (2000). Transnationalization in international migration: implications for the study of citizenship and culture. Ethnic and Racial Studies, 23, (2), 189-222. 
Feinberg, W. (1998). Common schools, uncommon identities: national unity and cultural difference. New Haven CT: Yale University Press.

Franklin, B. (Ed.). (1995). The handbook of children's rights. Comparative policy and practice. London: Routledge.

Freire, P. (1998). Pedagogy of Freedom: Ethics, Democracy, and Civic Courage. Lanham, Maryland \& Oxford: Rowman \& Littlefield Publishers.

Fullinwider, R. (2001). Multicultural education and cosmopolitan citizenship. International Journal of Educational Research, 35, 331-343.

Gellner, E. (1989). Nations et nationalisme. Paris: Payot.

Giddens, A. (1991). Modernity and self Identity: self and society in the late modern age. Oxford: Oxford University Press.

Gikandi, S. (1996). Maps of Englishness. New York: Columbia University Press.

Gordon, D.T. (2002). Teaching civics after September 11. Will the swell of patriotic expression translate into better civics teaching and learning in U.S. schools? Harvard Education Letter, January/February.

Grant, C.A. \& Ladson-Billings, G. (1997). Dictionary of multicultural education. Phoenix, AZ: Oryx Press.

Green, A. (1990). Education and state formation: The rise of education systems in England, France and the USA. London: Macmillan.

Griffith, R. (1998). Educational citizenship and independent learning. London: Jessica Kingsley Publishers.

Gwyn, R.(1995). Nationalism without walls: the unbearable lightness of being Canadian. Toronto: McClelland and Stewart.

Haire, C. \& Manley-Casimir, M. (2000). Restoring duty to discourse of rights and citizenship Education: a radical retrenchment? Encounters on Education, 1, [http://educ.queensu.ca I-eoelencounters_index.html].

Hahn, C. (1999). Citizenship education: an empirical study of policy, practices and outcomes. Oxford Review of Education. 25, (1), 231-250.

Han, C.L. (1998). Becoming political: comparative perspectives on citizenship education. New York: SUNY.

Hart, R.A. (1992). Children's participation: from tokenism to citizenship. Florence: UNICEF /International Child Development Centre.

Harwood, A.M. (1992). Classroom climate and civic education in secondary social studies research. Antecedents and findings. Theory and Research in Social Education, 20, 47-86.

Heater, D. (1990). Citizenship: the civic ideal in world history, politics and education. London: Longman.

Hébert, Y. (1997). Citizenship education: towards a pedagogy of social participation and identity formation. Canadian Ethnic Studies, XXIX, (2), 82-96.

Hirsch, E.D. (1987). Cultural Literacy: what every American needs to know. New York: Random House.

Hirsch, E.D. (1991). What Your 2nd grader needs to know: fundamental of a good second-grade education. New York: Dell.

Kaplan, W. (Ed.) (1993). Belonging: the meaning and future of Canadian citizenship. Montreal: McGill-Queen's University Press.

Kukathas, C. (2001). Education and citizenship in divers societies. International Journal of Educational Research, 35, 319-330.

Kymlicka, W. (1995a). Multicultural citizenship. Toronto: Oxford University Press.

Kymlicka, W. (1995b). The Rights of minority cultures. Toronto: Oxford University Press.

Kymlicka, W. \& Norman, W. (Ed.). (2000). Citizenship in diverse societies. Oxford: Oxford University Press.

Lister, R. (1990). The Exclusive Society: citizenship and the poor. London: Child Poverty Action Group. 
Lynch, D. (1992). Education for citizenship in a multicultural society. London: Cassel.

Mac an Ghaill, M. (1996). Sociology, state schooling and social class: beyond critiques of the New Right hegemony. British Journal of Sociology of Education, 17, (2), 163-176.

Macedo, S. (2000). Diversity and distrust: Civic education in a multicultural democracy. Cambridge, MA: Harvard University Press.

MCEETYA. (1999). The Adelaide declaration for national common and agreed goals for schooling [online], available from: http://www.curriculum.edu.au/mceetya.

McLaren, P. (1998). Revolutionary pedagogy in post-revolutionary times: rethinking the political economy of critical education. Educational Theory, 48, (4), 431-462.

McLaren, P. \& Farahmandpur, R. (2001). Revolutionary citizenship as a pedagogy of resitance. Educational Policy, 15, (3), 343-376.

Mehan, H., Lintz, A, Kamoto D., \& Wills, J.S. (1995). Ethnographic studies of multicultural education in classrooms and schools. In J. Banks \& C.M Banks (Ed.), Handbook on research in multicultural education (pp. 129-144). New York: Macmillan

Mercredi, O. \& Turpel, M.E. (1993). In the rapids. Toronto: Penguin Books.

Morse, S. W. (1993). The Practice of citizenship: Learn by doing. The Social Studies, 84, (4), 164-167.

National Council for Social Studies (1992). Curriculum guidelines for multicultural education. Social Education, 55, 274-294.

National Curriculum Council (1990). Education for citizenship: curriculum Guidance 8. York: National Curriculum Council.

New York State Social Studies Syllabus Review and Development Committee (1991). One nation, many peoples: a declaration of cultural interdependence. Albany, New York: Department of Education.

O'Brien, M. \& Parry, L. (2001). Forming a more perfect union: reconceptualizing civics and citizenship education in Australia. Paper presented at the International Assembly of the $\mathrm{Na}-$ tional Council for Social Studies, Friday November 2001, Washington, DC.

Ogbu, J.U. (1987). Ethnoecology of urban schooling. New York: Colombia University Press.

Osborne, K. (1996). Education is the best national insurance: citizenship education in Canadian schools past and present. Canadian and International Education, 25, (2), 31-58.

Pallas, A.M., Natriello, G. \& McDill, E.L. (1989). The changing nature of the disadvantaged population: current dimensions and future trends. Educational researcher, 18, (5), 16-22.

Patrick, J.J \& Hoge, J.D. (1991). Teaching government, civics and law. In J.P. Shaver (Ed.), Handbook of research on social studies teaching and learning (pp. 427-436). New York: Macmillan.

Parker, W. C. (1990). Assessing citizenship. Educational Leadership, 48, (3), 17-22.

Phillips, R. (1996). History teaching, cultural restorationism and national identity in England and Wales. Curriculum Studies, 4, (3), 385-399.

Print, M. (1996). The new civics education: an integrated approach for Australian schools. Social Education, 60, (7), 443-446.

Qualifications and Curriculum Authority (1998). Education for citizenship and the teaching of democracy in schools. London: Qualifications and Curriculum Authority.

Ranson, S. (1993). Markets or democracy for education. British Journal of Educational Studies, 41, (4), 333-352.

Said, E. (1993). Culture and imperialism. London: Vintage Books.

Scetlander (1986). Carrigan street. Glasgow: Scetlander.

Schlesinger, A.M. (1991). The Disuniting of America. Knoxville, Tenn: Whittle Direct Books.

Sears, A.M. \& Hughes, A.S. (1996). Citizenship education and current educational reform. Canadian Journal of Education, 21, (2), 123-142.

Sears, A. (1994). Social studies as citizenship education in english Canada: a review of research. Theory and Research in Social Education, 22, 6-43. 
Sears, A. (1996). Something Different to everyone: conceptions of citizenship and citizenship education. Canadian and International Education, 25, 2.

Sleeter, C. (1991). Empowerment through multicultural education. Albany, NY: State University of New York Press.

Starkey, H. (Ed.) (1991). The challenge of human rights education. London: Cassel.

Taylor, C. (1992). Rapprocher les solitudes: écrits sur la fédéralisme et le nationalisme au Canada. Sainte-Foy: Les Presses de l'Université Laval.

Tiedt, P.L. \& Tiedt, I.M (1990). Multicultural teaching: A handbook of activities, information and resources. Boston: Allyn and Bacon.

Torney-Purta, J., Schwille, J. \& Amadeo, J. (Ed.). (1999). Civic education across countries: twenty-four national case studies from the IEA Civic Education Project. Amsterdam: International Association for the Evaluation of Educational Achievement.

Torres, C.A. (1998). Education, democracy and multiculturalism: dilemmas of citizenship in a multicultural society. Ladham, Md.: Rowman and Littlefield.

Van Sledright, B.A. \& Grant, S.G. (1994). Citizenship education and the persistent nature of classroom teaching dilemmas. Theory and Research in Social Education, 22, 305-339.

U.S. Bureau of the Census (1994). Statistical abstract of the United States. Washington, D.C: Government Printing Office.

Webber, J. (1996). Multiculturalism and the limits to toleration. In Language, Culture and Values in Canada at the Dawn of the 21st Century/Langues, cultures et valeurs au Canada à l'aube du XXIe siècle (pp. 269-279). Ottawa: International Council for Canadian Studies/Carleton University Press.

White, J. (1991). The justification of autonomy as an educational aim. London: Cassel.

Womack, S.T. \& King, O.R. (1982). A Case for citizenship education in the early years. Durant, OK: Southeastern Oklahoma State University; Huntsville, TX: Sam Houston State University.

Zelman, G.L. (1975). Antidemocratic beliefs: a survey and some explanations. Journal of Social Issues, 31, 31-53.

\section{Multikulturelle Erziehung und politische Bildung: Haupttendenzen und aktuelle Debatten im angelsächsischen Sprachraum}

\section{Zusammenfassung}

Ausgehend von den angelsächsischen Forschungen der 1990er-Jahre analysiert dieser Beitrag die Zusammenhänge zwischen politischer Bildung und multikultureller Erziehung. Zunächst wird auf die institutionellen Wurzeln der politischen Bildung und der multikulturellen Erziehung in den angelsächsischen Staaten hingewiesen. Anschliessend wird eine Bilanz der Forschung zur politischen Bildung präsentiert. Zwei Themen dieser Forschung werden detaillierter angesprochen: die unterschiedlichen Typen der politischen Bildung und die Ansprüche der kulturellen Minderheiten. Abschliessend werden die Möglichkeiten einer Konvergenz zwischen multikultureller Erziehung und politischer Bildung geprüft. 


\section{Educazione multiculturale e educazione alla cittadinanza: dibattito e tendenze nel mondo anglosassone}

\section{Riassunto}

Partendo dalle ricerche effettuate nel mondo anglosassone negli anni '90, l'articolo analizza i legami tra educazione alla cittadinanza e educazione multiculturale. Dapprima si presentano alcune origini istituzionali dell'educazione alla cittadinanza e dell'educazione multiculturale per poi passare ad un bilancio delle ricerche. In particolare si approfondiscono due temi che appaiono in questi lavori anglosassoni: i differenti tipi di educazione alla cittadinanza e le rivendicazioni delle minoranze culturali. Per concludere si esaminano le possibilità di una convergenza tra educazione multiculturale e educazione alla cittadinanza.

\section{Multicultural education and citizen education: Principle tendencies and current debates in the English speaking world.}

\section{Summary}

This article analyzes the connections between citizen education and multicultural education based on English speaking research done in the 1990s. To begin with we will present several elements concerning the institutional roots of civic and multicultural education in English speaking countries. In a further section we will present the results of research done on citizen education. This will be done in a detailed manner covering two research themes in English speaking countries: the different types of citizen education and the challenges for minority cultures. Finally we will try to examine the possibilities of a convergence between multicultural education and citizen education. 